\title{
A monotone domain decomposition algorithm for nonlinear parabolic difference schemes in the canonical form
}

\author{
I. Boglaev ${ }^{1}$
}

(Received 2 August 2006; revised 6 September 2007)

\begin{abstract}
A monotone domain decomposition algorithm for a nonlinear algebraic system, which is a finite difference approximation of a nonlinear reaction-diffusion problem of parabolic type, is presented and is shown to converge monotonically either from above or from below to a solution of the system. The algorithm is based on a modification of the Schwarz alternating method and the method of upper and lower solutions. Advantages of the algorithm are that the algorithm solves only linear discrete systems at each iterative step, converges monotonically to the exact solution of the system, and is potentially parallelisable. Numerical experiments for a model problem from chemical engineering are presented.
\end{abstract}

See http://anziamj.austms.org.au/ojs/index.php/ANZIAMJ/article/view/67 for this article, (c) Austral. Mathematical Soc. 2007. Published September 29, 2007. ISSN 1446-8735 


\section{Contents}

1 Introduction

C398

2 Nonlinear difference scheme

C400

3 Monotone domain decomposition algorithm

C401

3.1 Statement of domain decomposition algorithm . . . . . . . C402

3.2 Monotone convergence of algorithm (8)-(11) . . . . . . . C404

4 Numerical experiments

C407

References

C411

\section{Introduction}

We are interested in nonlinear reaction-diffusion problems of parabolic type

$$
\begin{aligned}
& -\nabla \cdot(D \nabla U)+U_{t}=-f(x, t, U), \quad x \in \omega, \quad t>0 ; \\
& U(x, 0)=\psi(x), \quad x \in \bar{\omega} ; \\
& U(x, t)=g(x, t), \quad(x, t) \in \partial \omega \times(0, T] ; \\
& x \in \mathbb{R}^{k}, \quad \bar{\omega}=\prod_{\alpha=1}^{k} \bar{\omega}^{x_{\alpha}}, \quad \bar{\omega}^{x_{\alpha}}=\left\{0 \leq x_{\alpha} \leq r_{\alpha}\right\},
\end{aligned}
$$

where $\bar{\omega}=\omega \cup \partial \omega, \partial \omega$ is the boundary and $\nabla$ is gradient operator in $\omega$. We assume that $D=D(x, t)>0$ on $\bar{\omega} \times[0, T]$ for every $T<\infty$ and $D, f, \psi$ and $g$ are sufficiently smooth functions.

Difference schemes satisfying the maximum principle are called monotone. The monotonicity condition guarantees that systems of algebraic equations based on such methods are well-posed. A major point about the nonlinear monotone difference schemes is to obtain reliable and efficient computational 
algorithms for computing the solution. A fruitful method for the treatment of these nonlinear schemes is the method of upper and lower solutions [3]. Initial upper and lower solutions can be constructed directly from the difference equation without any knowledge of the solution. Thus, this approach simplifies considerably the search for the initial iteration as is often required in Newton's method.

Iterative domain decomposition algorithms based on Schwarz-type alternating procedures received much attention for their potential as efficient algorithms for parallel computing [5]. Boglaev [1], for solving two-dimensional nonlinear reaction-diffusion problems of parabolic type, proposed the discrete iterative algorithm which combines the monotone approach and the iterative domain decomposition method based on the Schwarz alternating procedure. The advantages of the algorithm are that the algorithm solves only linear discrete systems at each iterative step, converges monotonically to the exact solution of the system, and may be parallelised. The purpose of this article is to extend the monotone domain decomposition algorithm from Boglaev [1] on the nonlinear monotone difference schemes of parabolic type in the canonical form.

Section 2 presents the nonlinear monotone difference schemes in the canonical form and formulates the maximum principle. Section 3 constructs a monotone domain decomposition algorithm for solving the nonlinear schemes and proves its monotone convergence. The final Section 4 presents results of numerical experiments for a model problem from chemical engineering, where iteration counts and execution times between the monotone (undecomposed) iterative method and the monotone domain decomposition algorithm are compared. 


\section{Nonlinear difference scheme}

On $\bar{\omega}$ and $[0, T]$, we introduce rectangular meshes $\bar{\omega}^{h}$ and $\bar{\omega}^{\tau}$, respectively, and for simplicity assume that the mesh $\bar{\omega}^{\tau}$ is uniform with the time step $\tau$. For solving (1), we consider the nonlinear implicit difference scheme in the canonical form

$$
\begin{aligned}
& \mathcal{L} u(p, t)=-f(p, t, u)+\tau^{-1} u(p, t-\tau), \quad(p, t) \in \omega^{h} \times\left(\bar{\omega}^{\tau} \backslash\{0\}\right), \\
& u(p, 0)=\psi(p), \quad p \in \bar{\omega}^{h}, \\
& u(p, t)=g(p, t), \quad(p, t) \in \partial \omega^{h} \times\left(\bar{\omega}^{\tau} \backslash\{0\}\right),
\end{aligned}
$$

where $\bar{\omega}^{h}=\omega^{h} \cup \partial \omega^{h}, \partial \omega^{h}$ is the boundary, and the difference operator $\mathcal{L}$ is defined by

$\mathcal{L} u(p, t) \equiv\left(\mathcal{L}^{h}+\frac{1}{\tau}\right) u(p, t), \quad \mathcal{L}^{h} u(p, t) \equiv d(p, t) u(p, t)-\sum_{p^{\prime} \in \sigma^{\prime}(p)} a_{p}\left(p^{\prime}, t\right) u\left(p^{\prime}, t\right)$, where $\sigma^{\prime}(p)=\sigma(p) \backslash\{p\}, \sigma(p)$ is a stencil of the scheme.

On each time level $t$, we make the following assumptions on the coefficients of the spatial operator $\mathcal{L}^{h}$ :

$$
d(p, t)>0, \quad a_{p}\left(p^{\prime}, t\right) \geq 0, \quad d(p, t)-\sum_{p^{\prime} \in \sigma^{\prime}(p)} a_{p}\left(p^{\prime}, t\right) \geq 0,
$$

where $p \in \omega^{h}$ and $p^{\prime} \in \sigma^{\prime}(p)$.

On each time level $t$, introduce the linear problem

$$
(\mathcal{L}+c) w(p, t)=f_{0}(p, t), \quad p \in \omega^{h}, \quad w\left(\partial \omega^{h}, t\right)=g\left(\partial \omega^{h}, t\right),
$$

where $c(p, t) \geq c_{0}=$ const $\geq 0, p \in \bar{\omega}^{h}$. We now formulate the maximum principle for the difference operator $\mathcal{L}+c$ and give an estimate of the solution to $(4)$. 
Lemma 1 Let the coefficients of the difference operator $\mathcal{L}^{h}$ satisfy (3).

1. If a mesh function $w(p, t)$ satisfies the conditions

$$
(\mathcal{L}+c) w(p, t) \geq 0(\leq 0), \quad p \in \omega^{h}, \quad w\left(\partial \omega^{h}, t\right) \geq 0(\leq 0),
$$

then $w(p, t) \geq 0(\leq 0), p \in \bar{\omega}^{h}$.

2. The following estimate of the solution to (4) holds true

$$
\|w(t)\|_{\bar{\omega}^{h}} \leq \max \left\{\|g(t)\|_{\partial \omega^{h}},\left\|f_{0}(t)\right\|_{\omega^{h}} /\left(c_{0}+\tau^{-1}\right)\right\},
$$

where $\|g(t)\|_{\partial \omega^{h}} \equiv \max _{p \in \partial \omega^{h}}|g(p, t)|,\left\|f_{0}(t)\right\|_{\omega^{h}} \equiv \max _{p \in \omega^{h}}\left|f_{0}(p, t)\right|$.

The lemma is proved in the same manner as in Boglaev [1] for the case of two-dimensional problems.

\section{Monotone domain decomposition algorithm}

We assume that $f(x, t, U)$ from (1) satisfies the two sided constraints

$$
0 \leq f_{u} \leq c^{*}, \quad c^{*}=\mathrm{const}, \quad\left(f_{u}=\partial f / \partial U\right),
$$

where the assumption $f_{u} \geq 0$ is obtained via a change of variables.

On $\bar{\omega}^{x_{\alpha}}, \alpha=1, \ldots, k$, we set up nonuniform rectangular meshes

$$
\bar{\omega}^{h x_{\alpha}}=\left\{x_{\alpha}^{\left(i_{\alpha}\right)}, 0 \leq i_{\alpha} \leq N_{\alpha} ; x_{\alpha}^{(0)}=0, x_{\alpha}^{\left(N_{\alpha}\right)}=r_{\alpha}\right\}, \quad \alpha=1, \ldots, k .
$$

Thus we represent the mesh

$$
\bar{\omega}^{h}=\prod_{\alpha=1}^{k} \bar{\omega}^{h x_{\alpha}}, \quad \bar{\omega}^{h}=\omega^{h} \cup \partial \omega^{h},
$$


where $\omega^{h}$ and $\partial \omega^{h}$ are sets of interior and boundary mesh points, respectively.

By hyperplanes

$$
\left\{x_{1}=\rho_{m}, m=1, \ldots, M-1: \rho_{0}=0<\rho_{1}<\cdots<\rho_{M-1}<\rho_{M}=r_{1}\right\},
$$

we decompose the mesh $\bar{\omega}^{h}$ into $M$ nonoverlapping rectangular subdomains $\omega_{m}^{h}$, $m=1, \ldots, M$ :

$$
\begin{aligned}
& \bar{\omega}^{h}=\bigcup_{m=1}^{M} \bar{\omega}_{m}^{h}, \quad \partial \omega_{m}^{h}=\gamma_{m}^{0} \cup \gamma_{m-1} \cup \gamma_{m}, \quad \gamma_{m}^{0}=\partial \omega^{h} \cap \bar{\omega}_{m}^{h}, \\
& \gamma_{m}=\left\{\rho_{m}\right\} \times \bar{\omega}^{h y}, \quad \bar{\omega}^{h y} \equiv \prod_{\alpha=2}^{k} \bar{\omega}^{h x_{\alpha}}, \quad \bar{\omega}_{m}^{h} \cap \bar{\omega}_{m+1}^{h}=\gamma_{m},
\end{aligned}
$$

where the boundary $\partial \omega_{m}^{h}$ of $\bar{\omega}_{m}^{h}$ consists of the boundaries $\gamma_{m-1}, \gamma_{m}$ which belong to the hyperplanes $\rho_{m-1}$ and $\rho_{m}$, respectively, and $\gamma_{m}^{0}$ which belongs to the boundary $\partial \omega^{h}$. On $\bar{\omega}^{h}$ we introduce $(M-1)$ interfacial subdomains $\bar{\vartheta}_{m}^{h}$, $m=1, \ldots, M-1$, with boundaries $\partial \vartheta_{m}^{h}$ in the form

$$
\begin{aligned}
& \partial \vartheta_{m}^{h}=\gamma_{m}^{c} \cup \gamma_{m}^{b} \cup \gamma_{m}^{e}, \quad \gamma_{m}^{c}=\partial \omega^{h} \cap \bar{\vartheta}_{m}^{h}, \\
& \gamma_{m}^{b}=\left\{\rho_{m}^{b}\right\} \times \bar{\omega}^{h y}, \quad \gamma_{m}^{e}=\left\{\rho_{m}^{e}\right\} \times \bar{\omega}^{h y}, \quad \rho_{m}^{b}<\rho_{m}<\rho_{m}^{e},
\end{aligned}
$$

where $\bar{\vartheta}_{m}^{h}$ overlaps $\bar{\omega}_{m}^{h} \cup \bar{\omega}_{m+1}^{h}$. Figure 1 illustrates the domain decomposition in the two dimensional case $\mathbb{R}^{2}$.

We assume that sizes of all the subdomains $\bar{\omega}_{m}^{h}$ and $\bar{\vartheta}_{m}^{h}$ allow to solve Dirichlet boundary value problems based on the difference equation from (2).

\subsection{Statement of domain decomposition algorithm}

On each time level $t \in \bar{\omega}^{\tau} \backslash\{0\}$, we calculate iterates $v^{(n)}(p, t), p \in \bar{\omega}^{h}$ as follows. 


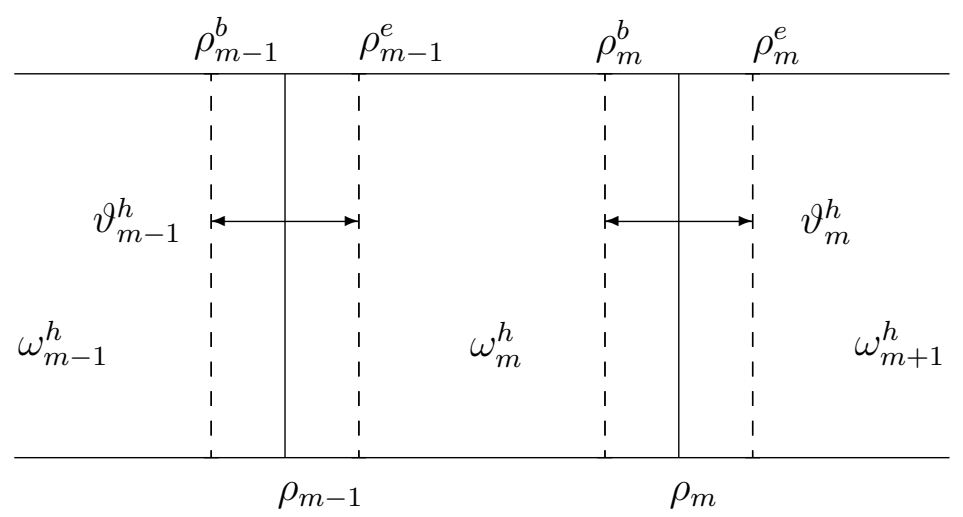

FiguRE 1: Fragment of the domain decomposition.

1. On the whole mesh $\bar{\omega}^{h}$, choose $v^{(0)}(p, t), p \in \bar{\omega}^{h}$ satisfying the boundary condition $v^{(0)}(p, t)=g(p, t)$ on $\partial \omega^{h}$.

Given $v^{(n-1)}(p, t)$, Steps 2 and 3 below generate $v^{(n)}(p, t)$.

2. For each main subdomain $\bar{\omega}_{m}^{h}$, solve the linear difference problem

$$
\begin{aligned}
& \left(\mathcal{L}+c^{*}\right) z_{m}^{(n)}(p, t)=-\mathcal{R}\left(p, t, v^{(n-1)}\right), \quad p \in \omega_{m}^{h}, \\
& \mathcal{R}\left(p, t, v^{(n-1)}\right) \equiv \mathcal{L} v^{(n-1)}(p, t)+f\left(p, t, v^{(n-1)}\right)-\tau^{-1} v(p, t-\tau),(8)
\end{aligned}
$$

with $z_{m}^{(n)}\left(\partial \omega_{m}^{h}, t\right)=0$, and $\mathcal{R}\left(p, t, v^{(n-1)}\right)$ is the residual of $(2)$ on $v^{(n-1)}$.

3. For each interfacial subdomain $\bar{\vartheta}_{m}^{h}$, solve the linear problem

$$
\left(\mathcal{L}+c^{*}\right) \tilde{z}_{m}^{(n)}(p, t)=-\mathcal{R}\left(p, t, v^{(n-1)}\right), \quad p \in \vartheta_{m}^{h},
$$

with $\tilde{z}_{m}^{(n)}\left(\partial \vartheta_{m}^{h}, t\right)$ defined by the mesh functions computed in Step 2. 
4. Compute the mesh function $v^{(n)}(p, t), p \in \bar{\omega}^{h}$, by piecing together the solutions on the subdomains

$$
v^{(n)}(p, t)= \begin{cases}v^{(n-1)}(p, t)+z_{m}^{(n)}(p, t), & p \in \bar{\omega}_{m}^{h} \backslash\left(\bar{\vartheta}_{m-1}^{h} \cup \bar{\vartheta}_{m}^{h}\right), \\ v^{(n-1)}(p, t)+\tilde{z}_{m}^{(n)}(p, t), & p \in \bar{\vartheta}_{m}^{h}\end{cases}
$$

5. If the stopping criterion

$$
\left\|\mathcal{R}\left(t, v^{(n)}\right)\right\|_{\omega^{h}} \leq \delta
$$

is reached, then stop and set up $v(p, t)=v^{\left(n_{*}\right)}(p, t), p \in \bar{\omega}^{h}$, otherwise go to Step 2, where $\delta$ is a prescribed accuracy and $n_{*}$ is minimal subject to (11).

Algorithm (8)-(11) can be carried out by parallel processing since on each iterative step $n$ the $M$ problems (8) and the $(M-1)$ problems (9) can be implemented concurrently.

\subsection{Monotone convergence of algorithm (8)-(11)}

On a time level $t \in \bar{\omega}^{\tau} \backslash\{0\}$, we say that $\bar{v}(p, t)$ is an upper solution with respect to a given function $v(p, t-\tau)$ if it satisfies

$$
\mathcal{L} \bar{v}(p, t)+f(p, t, \bar{v})-\tau^{-1} v(p, t-\tau) \geq 0, \quad p \in \omega^{h}, \quad \bar{v}\left(\partial \omega^{h}, t\right) \geq g\left(\partial \omega^{h}, t\right) .
$$

Similarly, $\underline{v}(p, t)$ is called a lower solution with respect to $v(p, t-\tau)$ if it satisfies the reversed inequalities. On each time level, upper and lower solutions satisfy the inequality

$$
\underline{v}(p, t) \leq \bar{v}(p, t), \quad p \in \bar{\omega}^{h} .
$$

We get the following convergence properties of algorithm (8)-(11). 
Theorem 2 Assume that the coefficients of the difference operator $\mathcal{L}$ in (2) satisfy (3) and $f(p, t, u)$ satisfies (6). Let $v(p, t-\tau)$ be given and $\bar{v}^{(0)}(p, t)$ and $\underline{v}^{(0)}(p, t)$ be upper and lower solutions corresponding to $v(p, t-\tau)$. Then the upper sequence $\left\{\bar{v}^{(n)}(p, t)\right\}$ generated by (8)-(11) converges monotonically from above to the unique solution $v(p, t)$ of the problem

$$
\mathcal{L} v(p, t)+f(p, t, v)-\tau^{-1} v(p, t-\tau)=0, \quad p \in \omega^{h},
$$

and the lower sequence $\left\{\underline{v}^{(n)}(p, t)\right\}$ generated by (8)-(11) converges monotonically from below to $v(p, t)$.

Proof: We consider only the case of the upper sequence. Let $\bar{v}^{(n-1)}$ be an upper solution. By the maximum principle in Lemma 1, from (8) we conclude that

$$
z_{m}^{(n)}(p, t) \leq 0, \quad p \in \bar{\omega}_{m}^{h}, \quad m=1, \ldots, M .
$$

From here, by the mean-value theorem and (6), we obtain from (8)

$$
\begin{aligned}
& \mathcal{R}\left(p, t, v_{m}^{(n)}\right)=-\left(c^{*}-f_{u}^{(n)}(p, t)\right) z_{m}^{(n)}(p, t) \geq 0, \quad p \in \omega_{m}^{h} \\
& v_{m}^{(n)}(p, t)=\bar{v}^{(n-1)}(p, t), \quad p \in \partial \omega_{m}^{h} \\
& v_{m}^{(n)}(p, t)=\bar{v}^{(n-1)}(p, t)+z_{m}^{(n)}(p, t)
\end{aligned}
$$

Taking into account (14) and that $\bar{v}^{(n-1)}$ is an upper solution, by the maximum principle in Lemma 1, from (9) it follows that

$$
\tilde{z}_{m}^{(n)}(p, t) \leq 0, \quad p \in \bar{\vartheta}_{m}^{h}, \quad m=1, \ldots, M-1 .
$$

Similarly, from (9) we obtain the difference problem for $\tilde{v}_{m}^{(n)}=\bar{v}^{(n-1)}+\tilde{z}_{m}^{(n)}$

$$
\mathcal{R}\left(p, t, \tilde{v}_{m}^{(n)}\right)=-\left(c^{*}-f_{u}^{(n)}(p, t)\right) \tilde{z}_{m}^{(n)}(p, t) \geq 0, \quad p \in \vartheta_{m}^{h},
$$

with the boundary conditions $\tilde{v}_{m}^{(n)}\left(\gamma_{m}^{c}, t\right)=g\left(\gamma_{m}^{c}, t\right), \tilde{v}_{m}^{(n)}\left(\gamma_{m}^{b}, t\right)=v_{m}^{(n)}\left(\gamma_{m}^{b}, t\right)$ and $\tilde{v}_{m}^{(n)}\left(\gamma_{m}^{e}, t\right)=v_{m+1}^{(n)}\left(\gamma_{m}^{e}, t\right)$. Now from here, (15) and the definition of $v^{(n)}$ 
in (10), we conclude that

$$
\mathcal{R}\left(p, t, v^{(n)}\right) \geq 0, \quad p \in \omega^{h} \backslash \bigcup_{m=1}^{M-1} \gamma_{m}^{b, e} .
$$

From the boundary conditions for $v_{m}^{(n)}$ and $\tilde{v}_{m}^{(n)}$, it follows that $v^{(n)}$ satisfies the boundary condition in (2). Thus, to prove that $v^{(n)}$ is an upper solution of problem (2), we have to verify that the last inequality holds true on the interfacial boundaries $\gamma_{m}^{b}$ and $\gamma_{m}^{e}, m=1, \ldots, M-1$. We check this inequality in the case of the left interfacial boundary $\gamma_{m}^{b}$, since the second case is checked in a similar way. From (8), (9), (14) and (16), we conclude that the mesh function $w_{m}^{(n)}=v_{m}^{(n)}-\tilde{v}_{m}^{(n)}$ satisfies the difference problem

$$
\begin{aligned}
& \left(\mathcal{L}+c^{*}\right) w_{m}^{(n)}(p, t)=0, \quad p \in \vartheta_{m}^{h b}=\omega_{m}^{h} \cap \vartheta_{m}^{h}, \\
& w_{m}^{(n)}(p, t)=0, \quad p \in \partial \vartheta_{m}^{h b} \backslash \gamma_{m}, \quad w_{m}^{(n)}(p, t) \geq 0, \quad p \in \gamma_{m} .
\end{aligned}
$$

In view of the maximum principle in Lemma $1, v_{m}^{(n)}(p, t)-\tilde{v}_{m}^{(n)}(p, t) \geq 0$, $p \in \bar{\vartheta}_{m}^{h b}$. From here, (3), (10) and (15), and taking into account $\tilde{v}_{m}^{(n)}\left(\gamma_{m}^{b}, t\right)=$ $v_{m}^{(n)}\left(\gamma_{m}^{b}, t\right)$, it follows that

$$
\mathcal{R}\left(p, t, v^{(n)}\right) \geq \mathcal{R}\left(p, t, v_{m}^{(n)}\right) \geq 0, \quad p \in \gamma_{m}^{b} .
$$

This leads to the fact that $v^{(n)}$ is an upper solution of problem (13).

For arbitrary $p \in \omega^{h}$, it follows from (14), (16) and (12) that the sequence $\left\{\bar{v}^{(n)}(p, t)\right\}$ is monotonically decreasing and bounded below by $\underline{v}(p, t)$, where $\underline{v}$ is any lower solution. Therefore, the sequence is convergent and it follows from (8) and (9) that $\lim z_{m}^{(n)}(p, t)=0$ and $\lim \tilde{z}_{m}^{(n)}(p, t)=0$ as $n \rightarrow \infty$. Now by linearity of the operator $\mathcal{L}$ and the continuity of $f$, we have also from (8) and (9) that the mesh function $v$ defined by $v(p, t)=\lim _{n \rightarrow \infty} \bar{v}^{(n)}(p, t)$, $p \in \bar{\omega}^{h}$, is an exact solution to (13). The uniqueness of the solution to (13) follows from the maximum principle in Lemma 1 and (6). This proves the theorem. 
Theorem 3 Let the coefficients of the difference operator $\mathcal{L}$ in (2) satisfy (3), $f(p, t, u)$ satisfy (6), and $v^{(0)}(p, t)$ be an upper or lower solution. If on each time level the stopping criterion (11) is satisfied, then for the monotone domain decomposition algorithm (8)-(11), the following estimate holds:

$$
\max _{t \in \bar{\omega}^{\tau}}\|v(t)-u(t)\|_{\bar{\omega}^{h}} \leq \delta T,
$$

where $u(p, t)$ is the solution to (2). Furthermore, on each time level the sequence $\left\{v^{(n)}(p, t)\right\}$ converges monotonically.

Proof: The difference problem for $v(p, t)=v^{\left(n_{*}\right)}(p, t)$ is

$$
\mathcal{L} v(p, t)+f(p, t, v)-\tau^{-1} v(p, t-\tau)=\mathcal{R}\left(p, t, v^{\left(n_{*}\right)}\right), \quad p \in \omega^{h},
$$

with the boundary condition $v\left(\partial \omega^{h}, t\right)=g\left(\partial \omega^{h}, t\right)$. From here, (2) and using the mean-value theorem, we get a difference problem for $w(p, t)=v(p, t)-$ $u(p, t)$ :

$\left(\mathcal{L}+f_{u}^{\left(n_{*}\right)}\right) w(p, t)=\mathcal{R}\left(p, t, v^{\left(n_{*}\right)}\right)+\tau^{-1} w(p, t-\tau), \quad p \in \omega^{h}, \quad w\left(\partial \omega^{h}, t\right)=0$.

From here, using (5) and taking into account that according to Theorem 2, the stopping criterion (11) is always satisfied, we have

$$
\|w(t)\|_{\bar{\omega}^{h}} \leq \tau\left\|\mathcal{R}\left(t, v^{\left(n_{*}\right)}\right)\right\|_{\omega^{h}}+\|w(t-\tau)\|_{\bar{\omega}^{h}} \leq \tau \delta+\|w(t-\tau)\|_{\bar{\omega}^{h}} .
$$

Since $w(p, t)=0$, we conclude that $\|w(t)\|_{\bar{\omega}^{h}} \leq \delta T, t \in \bar{\omega}^{\tau}$. Thus, we prove the theorem.

\section{Numerical experiments}

We apply the monotone domain decomposition algorithm (8)-(11) to the nonlinear singularly perturbed parabolic problem

$$
-\mu^{2}\left(\frac{\partial^{2} U}{\partial x_{1}^{2}}+\frac{\partial^{2} U}{\partial x_{2}^{2}}\right)+\frac{\partial U}{\partial t}=-\frac{U-4}{5-U}, \quad\left(x_{1}, x_{2}, t\right) \in \omega \times(0, T],
$$




$$
\begin{aligned}
& U\left(x_{1}, x_{2}, 0\right)=0, \quad\left(x_{1}, x_{2}\right) \in \bar{\omega}, \\
& U\left(x_{1}, x_{2}, t\right)=1, \quad\left(x_{1}, x_{2}\right) \in \partial \omega \times(0, T], \\
& \omega=\left\{0<x_{1}<1\right\} \times\left\{0<x_{2}<1\right\},
\end{aligned}
$$

which models the biological Michaelis-Menten process without inhibition [2], where $\mu$ is a small positive parameter. The solution to the reduced elliptic problem $(\mu=0)$ is $U_{r}=4$. For $\mu \ll 1$ the problem is singularly perturbed [1] and the solution increases sharply from $U=1$ on $\partial \omega$ to $U=4$ on the interior. The solution to the parabolic problem approaches this steady state with time.

For the spatial differential operator, we use the central difference approximation on the five point rectangular stencil, which satisfies (3). We employ a layer adapted mesh of a piecewise uniform type [1]. If the parameter $\mu$ is small enough, then the uniform mesh inside of the boundary layers is fine, and the uniform mesh outside of the boundary layers is coarse. The central difference scheme on the piecewise uniform mesh converges $\mu$-uniformly to the solution of the test problem. We can show that $f_{u}$ is bounded above and below by $c^{*}=1$ and $c_{*}=1 / 25$ respectively.

We solve all linear systems in the monotone domain decomposition algorithm (8)-(11) with the restarted GMRES algorithm [4]. All experiments were performed on a serial computer equipped with a $2.8 \mathrm{GHz}$ Pentium 4 processor.

We consider balanced domain decompositions. A balanced domain decomposition is one in which the mesh points are equally distributed among the main subdomains. For balanced decompositions, the first and last interfacial subdomains each overlap the boundary layers. For a parallel implementation of the algorithm, balanced domain decompositions guarantee load balancing of computational nodes.

For $\mu \leq 10^{-2}$ the average convergence iteration counts over ten time levels and execution times are shown in Table 1. All execution times are rounded to the nearest second. Where there is some choice for the interfacial subdomain 
TABLE 1: Average convergence iteration counts and execution times with balanced domain decompositions.

\begin{tabular}{|c|c|c|c|c|c|c|c|c|c|c|c|c|c|c|c|}
\hline$\mu$ & \multicolumn{5}{|c|}{$10^{-2}$} & \multicolumn{5}{|c|}{$10^{-3}$} & \multicolumn{5}{|c|}{$10^{-4}$} \\
\hline \multirow{2}{*}{$N \backslash M$} & 1 & 4 & 8 & 16 & 32 & 1 & 4 & 8 & 16 & 32 & 1 & 4 & 8 & 16 & 32 \\
\hline & \multicolumn{15}{|c|}{ vergence iteration count } \\
\hline $2^{7}$ & 5.0 & $\frac{6.0}{50}$ & $\frac{6.0}{50}$ & $\frac{6.0}{50}$ & $\frac{6.0}{52}$ & 5.0 & $\frac{6.0}{50}$ & $\frac{6.0}{50}$ & $\frac{6.0}{50}$ & $\frac{6.0}{5 .}$ & 5.0 & $\frac{6.0}{50}$ & $\frac{6.0}{50}$ & $\frac{6.0}{50}$ & $\frac{6.0}{52}$ \\
\hline $2^{8}$ & 5.0 & $\frac{8.0}{50}$ & $\frac{8.0}{50}$ & $\frac{8.0}{50}$ & $\frac{8.0}{50}$ & 5.0 & $\frac{6.0}{50}$ & $\frac{7.6}{50}$ & $\frac{7.6}{50}$ & $\frac{7.7}{50}$ & 5.0 & 3.0 & $\frac{7.6}{50}$ & $\frac{7.6}{50}$ & 7.7 \\
\hline \multirow[t]{3}{*}{2} & 50 & 13.0 & 13.0 & 13.0 & 13 & 5 & 7.0 & 11.0 & 11.0 & & 50 & 6.0 & 11.0 & 11.0 & 11. \\
\hline & & 5.0 & 5.0 & 5.0 & & & 5.0 & 5.0 & 5.0 & 5.0 & & 5.0 & 5.0 & 5.0 & 5.6 \\
\hline & \multicolumn{15}{|c|}{ execution time (s) } \\
\hline $2^{7}$ & 1 & $\frac{1}{1}$ & $\frac{1}{1}$ & $\frac{1}{1}$ & $\frac{1}{1}$ & 1 & & & & $\frac{1}{2}$ & & & 1 & & \\
\hline $2^{8}$ & 7 & $\frac{10}{10}$ & $\frac{9}{1}$ & $\frac{7}{8}$ & $\frac{6}{7}$ & 9 & 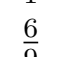 & $\frac{1}{8}$ & $\overline{7}$ & & & $\frac{0}{8}$ & $\frac{7}{9}$ & $\frac{6}{7}$ & $\frac{5}{6}$ \\
\hline $2^{9}$ & 60 & 220 & 196 & 131 & 92 & 67 & & 110 & 76 & 63 & & 72 & 110 & 78 & 63 \\
\hline & 60 & 147 & $\overline{142}$ & 96 & 74 & 67 & $\overline{101}$ & 98 & $\overline{69}$ & & 66 & 103 & $\overline{97}$ & $\overline{75}$ & 59 \\
\hline
\end{tabular}

widths, the results corresponding to minimal and maximal choices are written above and below the line, respectively. The convergence iteration count for each undecomposed problem with $M=1$ is 5.0 . If maximal interfacial subdomain widths are chosen, for $N=2^{7}$, the iteration count increases only slightly with decomposition and for $N \geq 2^{8}$, the iteration count for each decomposition is the same as for the undecomposed problem with $M=1$. Consider now the corresponding execution times. For $\mu \leq 10^{-3}$ the execution times of Table 1 demonstrate that, for each value of $N$ the decomposition $M=32$ with maximal interfacial subdomains reduces the execution time.

We now consider unbalanced domain decompositions with the interfacial subdomains located outside the boundary layers. All unbalanced domain decomposition experiments employ minimal interfacial subdomains. Average convergence iteration counts over ten time levels and execution times are shown in Table 2. For $\mu=10^{-2}$ the results are similar to those of the corresponding balanced decompositions with minimal interfacial subdomains. A comparison between the execution times of Table 1 and those of 
TABLE 2: Average convergence iteration counts and execution times with unbalanced domain decompositions.

\begin{tabular}{|c|c|c|c|c|c|c|c|c|c|c|c|c|c|c|c|}
\hline$\mu$ & \multicolumn{5}{|c|}{$10^{-2}$} & \multicolumn{5}{|c|}{$10^{-3}$} & \multicolumn{5}{|c|}{$10^{-4}$} \\
\hline$J \backslash M$ & 1 & 4 & 8 & 16 & 32 & 1 & 4 & 8 & 16 & 32 & & 4 & 8 & 16 & 32 \\
\hline & \multicolumn{15}{|c|}{ convergence iteration count } \\
\hline $2^{7}$ & 5 & 6 & 6 & 6 & 7 & 5 & 5 & 5 & 5 & 5 & 5 & 5 & 5 & 5 & 5 \\
\hline $2^{8}$ & 5 & 8 & 8 & 8 & 8 & 5 & 5 & 5 & 5 & 5 & 5 & 5 & 5 & 5 & 5 \\
\hline $2^{9}$ & 5 & 13 & 13 & 13 & 13 & 5 & 5 & 5 & 5 & 5 & 5 & 5 & 5 & 5 & 5 \\
\hline & \multicolumn{15}{|c|}{ execution time $(\mathrm{s})$} \\
\hline $2^{7}$ & 1 & 1 & 1 & 1 & 1 & 1 & 1 & 1 & 1 & 1 & & 1 & 1 & 1 & 1 \\
\hline $2^{8}$ & 7 & 10 & 9 & 8 & 7 & 9 & 5 & 5 & 5 & 5 & 9 & 6 & 5 & 5 & 5 \\
\hline $2^{9}$ & 60 & 221 & 198 & 158 & 145 & 67 & 61 & 56 & 53 & 51 & 67 & 61 & 57 & 53 & 51 \\
\hline
\end{tabular}

Table 2 shows that for $\mu \leq 10^{-3}$ the algorithm executes more quickly with unbalanced domain decomposition. For a parallel implementation of the algorithm with unbalanced domain decompositions, load balancing could be partially restored by solving the larger linear problems in parallel.

We draw the following conclusions with regard to the monotone domain decomposition algorithm (8)-(11).

- For all values of $\mu$ and $N$, and all domain decompositions, the convergence to the exact solution of the nonlinear difference scheme is monotonic.

- For $\mu \leq 10^{-3}$ the convergence iteration count is uniform with respect to $\mu$.

- For $\mu \leq 10^{-3}$ there are certain domain decompositions under which the algorithm executes more quickly than the undecomposed monotone algorithm with $M=1$. 
- For $\mu \leq 10^{-3}$ the algorithm executes more quickly when the domain decomposition is unbalanced rather than balanced.

Acknowledgements: The author is very grateful to Dr Matthew Hardy for his help with the implementation of the numerical experiments.

\section{References}

[1] I. Boglaev. Monotone iterative algorithms for a nonlinear singularly perturbed parabolic problem. J. Comp. Appl. Math., 172:313-335, 2004. doi:10.1016/j.cam.2004.02.010 C399, C401, C408

[2] E. Bohl. Finite Modelle gewöhnlicker Randwertaufgaben. Teubner, Stuttgart, 1981. C408

[3] C. V. Pao. Monotone iterative methods for finite difference system of reaction-diffusion equations. Numer. Math., 46:571-586, 1985. doi:10.1007/BF01389659 C399

[4] Y. Saad and M. H. Schultz. GMRES: A generalized minimal residual method for solving nonsymmetric linear systems. SIAM J. Sci .Stat. Comput., 7:856-869, 1986.

http://locus.siam.org/SISC/volume-07/art-0907058.html C408

[5] B. Smith, P. Bjørstad, and W. Gropp. Domain decomposition.

Cambridge University Press, Cambridge, 1996.

http://www. cambridge.org/0521602866 C399 


\section{Author address}

1. I. Boglaev, Institute of Fundamental Sciences, Massey University, Palmerston North, New Zealand.

mailto: i.boglaev@massey.ac.nz 